\title{
Initiating an Effective Personnel Development Program
}

\author{
Virginia A. Baldwin, William J. Gibbs, and \\ Marlene M. Slough
}

This study identified the educational interests of library personnel and investigated whether level of interest was a valid indicator of program participation. In the fall semester of 1993, Eastern Illinois University's Booth Library surveyed all administrators, faculty, and staff. The objectives of the survey were to identify: (1) indicators of educational interests of library personnel at a medium-sized university; (2) departmental differences in educational interests; and (3) the relationship between employee interest in educational programs and employee participation in programs developed.

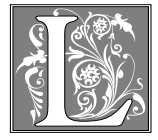

ibraries are constantly changing, and the personnel who work in them must be adaptable to keep pace in this fluid environment. Academic libraries have undergone transformations with respect to operating methods and the information services offered to patrons. Computers, mass-storage technology, and telecommunications have contributed significantly to these changes. ${ }^{1}$ Technological change has accelerated organizational change, and many professions, including librarianship, have responded by embracing continuing education. For example, it has been common for libraries to provide personnel development and training activities such as discussion groups and seminars on information technology or management development. ${ }^{2}$ There is an increased need for library administrators to build and maintain an atmosphere that fosters organizational development. ${ }^{3}$ One reason for the emphasis on human resource development is that well-trained and highly skilled personnel provide a competitive advantage in developing high-quality library programs. ${ }^{4}$ Because valuing human resources is viewed as essential to organizational excellence, those wanting a competitive edge must examine factors such as team-building, organizational culture, and people as resources. ${ }^{5}$ Organizations that can effectively develop human resources will likely achieve and maintain an advantage. ${ }^{6}$

Effective human resource development is a complex process. Personnel development programs frequently consist of workshops, demonstrations, or seminars; and, in and of themselves, these activities, although helpful, may not

Virginia A. Baldwin is an Associate Professor at Eastern Illinois University (EIU); e-mail: cfoab@eiu.edu. William J. Gibbs is an Associate Professor at EIU; e-mail: cfwig1@eiu.edu. Marlene M. Slough is an Instructor at EIU; e-mail: cfmms@eiu.edu. 


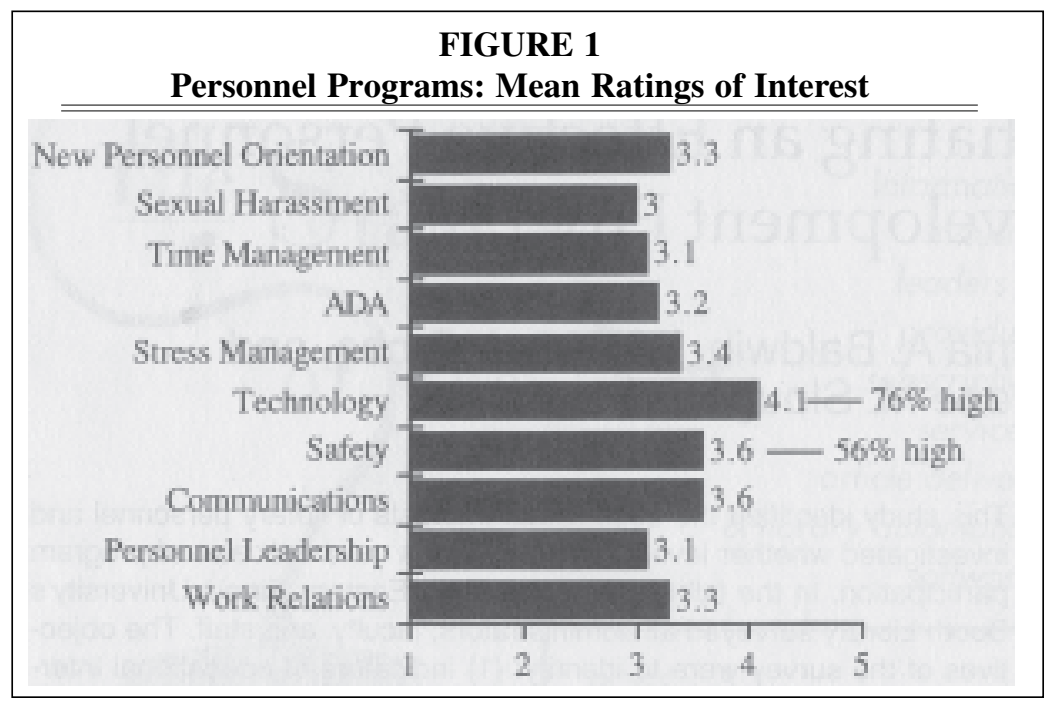

be solutions. Workshops and seminars frequently disseminate previously known information, ${ }^{7}$ or the information presented never gets transferred to the actual work situation. There are many reasons for their ineffectiveness, not the least of which is that often the activity's content, objectives, and mode of presentation are incongruent with participants' informational and learning needs. To be effective, developmental programs must be consistent with actual personnel needs and interests, and attendance must be maximized. Whether personnel make valuable transfer of what they learned to their work situation was not the focus of this study.

\section{Professional Development Study at Eastern Illinois University}

Eastern Illinois University, founded in 1895, has an enrollment of approximately 10,000 students. In the fall of 1993, Booth Library formed a new Personnel Development Committee. With the intention of increasing the effectiveness of its human resources by making personnel more knowledgeable, skilled, involved, and, ultimately, more resourceful, the committee rewrote its charge: "to identify areas of need and interest among Booth Library personnel and to develop and produce a continuing series of activities designed to enhance their skills and abilities." The committee did not want to begin offering workshops or training seminars without first determining the areas in which library personnel wanted and needed development.

A challenge Booth Library faced, along with many organizations, was to devise a method for identifying the developmental needs of an organization

The committee did not want to begin offering workshops or training seminars without first determining the areas in which library personnel wanted and needed development.

(in this case, a library) that has so many diversified services. ${ }^{8}$ The personnel committee eventually decided that surveying Booth personnel would be an approach that could yield worthwhile results. This approach is consistent with the Readiness, Planning, Training, Implementations, and Maintenance model (RPTIM) suggestion that "staff 


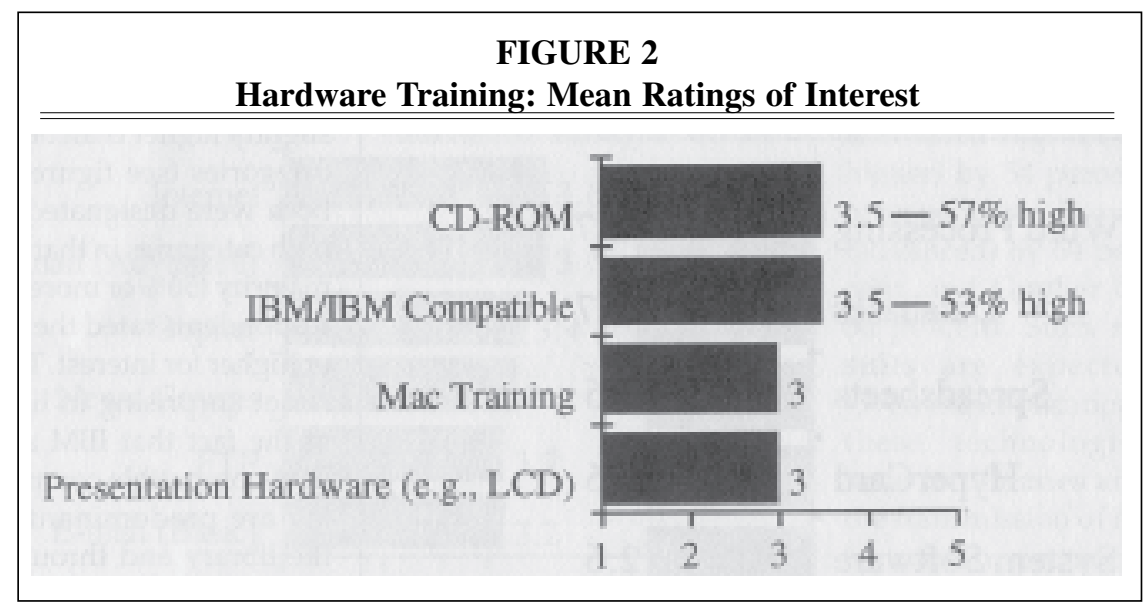

should be closely involved in the planning and selection of a program."

In addition, the survey was designed so that the following could be evaluated:

1. What relationship exists between personnel interest in a topic and personnel willingness to participate in a given program about that topic?

2. Are there departmental differences in personnel needs and interests?

\section{Methodology}

In the fall semester of 1993, the Personnel Development Committee requested that library administrators, faculty, and staff notify the committee in verbal or written form as to perceived organizational needs and to indicate activities that would help to alleviate those needs. Based on these responses, the committee created a survey instrument.

The survey presented thirty-nine potential categories of developmental activities. On a 5-point Likert-type scale, ordered from "No interest" to "Great idea," respondents rated their level of interest in the listed items and also indicated (yes or no) whether they would participate in a personnel development activity related to the topic. The survey also included open-ended questions and spaces for comments, and asked re- spondents to indicate the library department in which they worked.

The survey was given to a total of sixty-seven library personnel (administrators, faculty, and staff), and fortyeight usable responses were received. The thirty-nine categories were grouped into three areas: Personnel Programs (ten topics), Library and Electronic Resources (twenty-one topics), and Special Interest (eight topics). Ten library departments were surveyed.

\section{Results and Discussion}

The committee focused initially on those categories for which the majority of respondents (50\% or more) rated 4 or higher on the 5-point scale. These are hereafter referred to as high categories. This was done to expedite program development and to ensure that programming was consistent with personnel interests. Mean ratings were calculated for each category.

\section{Personnel Programs}

Personnel Programs consisted of ten categories of personnel development. Respondents rated from 1 to 5 the degree of interest they had for each category (see figure 1). As figure 1 indicates, respondents rated Technology highest. By the committee's definition 


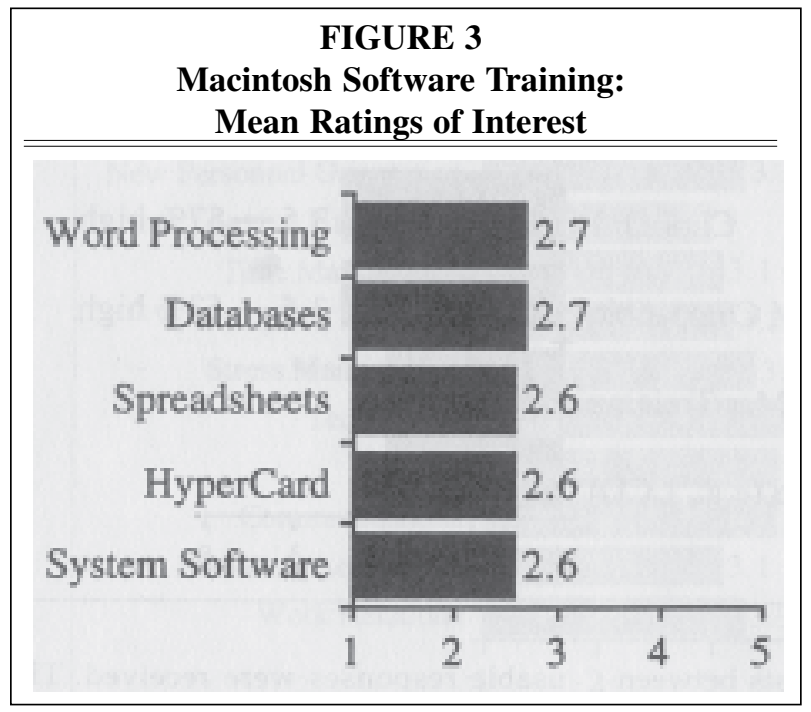

ries related to Library and Electronic Resources, each of which contained several items: Hardware Training, Macintosh Software Training, IBM/IBMCompatible Software Training, and Electronic Mail and Internet.

Hardware Training. For items related to hardware, respondents rated IBM/IBM-Compatible and CD-ROM slightly higher than other categories (see figure 2). Both were designated as high categories in that

of high, Technology and Safety and Emergency Procedures were the only two high categories under Personnel Programs. A majority (76\%) gave Technology high ratings whereas 56 percent of respondents rated Safety and Emergency Procedures as high. Communications among Library Personnel, although not a high category, received high ratings by 49 percent of respondents.

To some extent, all categories were valuable to respondents, but Technology generated the most interest. This is by no means surprising considering that libraries are in a constant state of technological flux and that those who work in this environment are forced to keep abreast of such changes. ${ }^{10}$

\section{Library and Electronic Resources}

The survey presented four general catego- the majority ( $50 \%$ or more) of respondents rated them 4 or higher for interest. This is not surprising in light of the fact that IBM and IBM-compatible computers are predominant in the library and throughout the university. Although differences between these categories are not great, this finding does suggest that employees may have a greater interest in activities that have applications to their job. In further support of this conclusion is the fact that the only li-

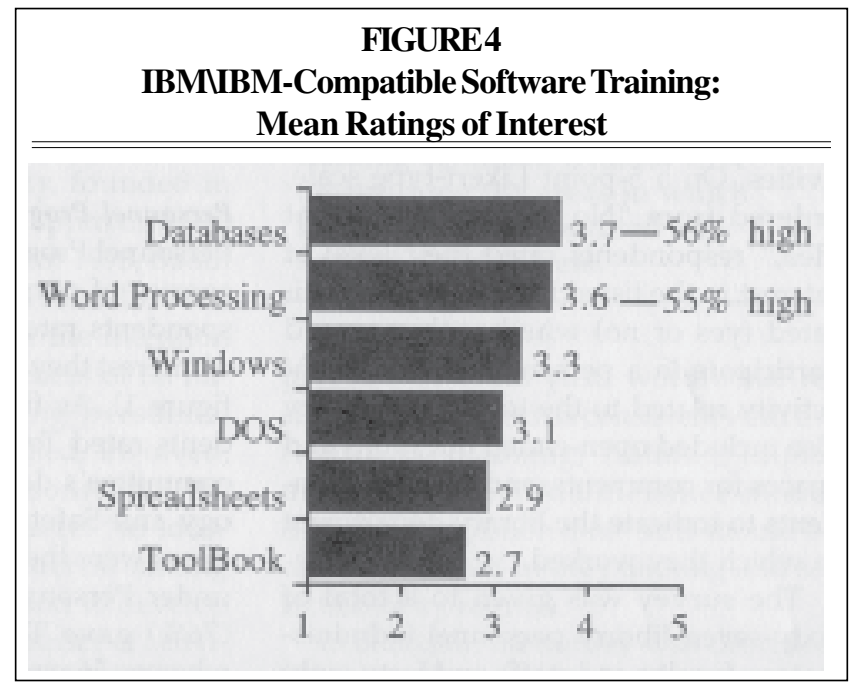




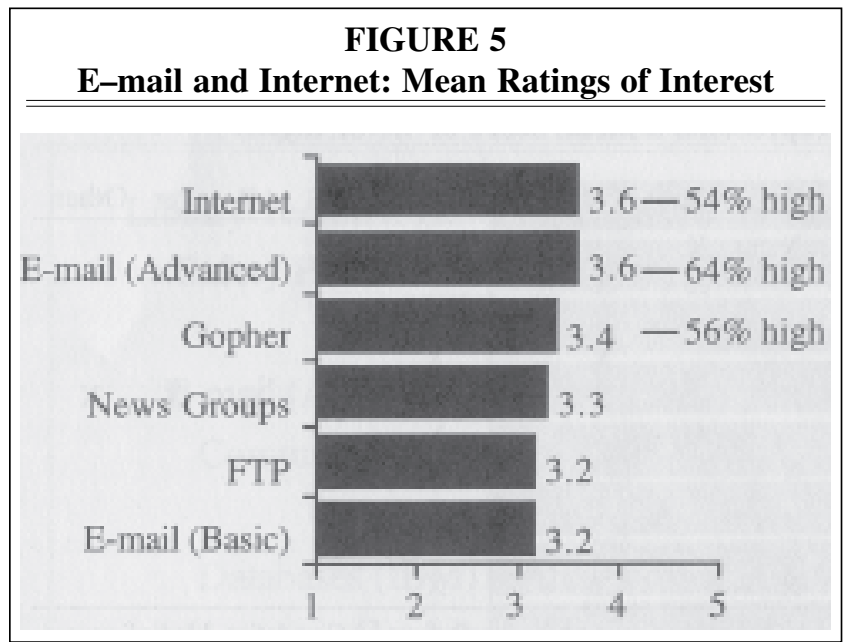

ware Training, again, a larger percentage of respondents gave a high-interest rating $(4$ or higher) to IBM/IBMcompatible than to Macintosh software. Of this group, only Word Processing and Databases fit the criterion to be designated as high.

E-mail and Internet. There were six categories related to $\mathrm{E}$ mail and the Internet, and all had mean interest ratings in the

brary department that indicated a relatively high interest in Macintosh training was Media Services, which uses Macintosh as the preferred computer platform.

Macintosh Software Training. Under Macintosh Software Training, the survey presented five categories (see figure 3 ). All received mean interest ratings of less than 3, and low percentages of respondents rated their interest in them as 4 or higher. No categories in this group were designated as high. However, taken by library departments, both Administration and Media Services indicated a high interest in these categories.

IBM/IBM-Compatible Software Training. Six categories were listed under IBM/ IBM-Compatible Software (see figure 4). Word Processing and Databases received the highest mean rating whereas responses to all other items were in the moderate range. For Soft- medium (3) range (see figure 5). The Internet was rated high (4 or higher) by 54 percent of respondents, E-mail (advanced) by 64 percent, and Gopher by 56 percent. Such results are expected given the implications these technologies have for libraries and the transmission of information electronically.

\section{Special-Interest Areas}

There were eight special-interest categories (see figure 6). The mean rating of interest for the categories in this group, like those for Macintosh software

\section{FIGURE 6}

\section{Special Interest Areas: Mean Ratings of Interest}

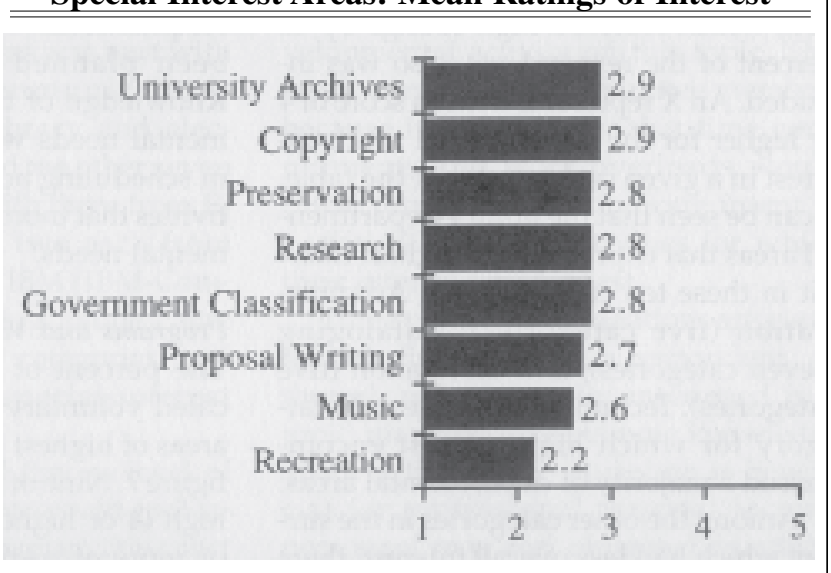




\begin{tabular}{|c|c|c|c|c|c|c|c|c|c|}
\hline \multicolumn{10}{|c|}{$\begin{array}{c}\text { TABLE } 1 \\
\text { Survey Results by Departmental Area } \\
\text { (where X represents a mean score of } 4.0 \text { or higher) } \\
\end{array}$} \\
\hline & Adm & Acq & Cat & Ref & Circ & MS & $\mathrm{AR}$ & Per & Other \\
\hline Communications & $X$ & $X$ & & & & & & & \\
\hline Internet & & $\mathrm{X}$ & $\mathrm{X}$ & & & & & & \\
\hline E-mail & & & $X$ & $\mathrm{X}$ & & & & & \\
\hline Safety & & & $X$ & & $X$ & & & & \\
\hline Technology & $X$ & & $\mathrm{X}$ & $\mathrm{X}$ & $\mathrm{X}$ & $X$ & $\mathrm{X}$ & $\mathrm{X}$ & $\mathrm{X}$ \\
\hline IBM Word Processing & & & $X$ & & $\mathrm{X}$ & & & & \\
\hline IBM Databases & $\mathrm{X}$ & & & & $\mathrm{X}$ & & & & \\
\hline Gopher & & & & & & $\mathrm{X}$ & & & \\
\hline CD-ROM & $\mathrm{X}$ & & $X$ & & & $X$ & & & \\
\hline IBM Hardware & $\mathrm{X}$ & & $X$ & & $\mathrm{X}$ & & & & \\
\hline
\end{tabular}

training, were all under 3 , and, similarly, a low percentage (less than $50 \%$ ) of respondents gave these categories a rating of 4 or 5 . Special Interest, as befits the name of this group, appeared to be the most department-specific group of categories, which accounts for the low overall mean. This will be discussed in more detail in the following section. None of the categories in this group was rated high, and the percentages of respondents with a 4 or 5 ranged from 14 percent for Recreation to 38 percent for Copyright.

\section{Survey Results by Departmental Area}

To determine whether there was a variation which would show that different areas of the library have different needs, survey respondents were asked which library department (area) he or she represents. Survey results by departmental area are displayed in table 1 for the nine categories (areas of interest) for which 50 percent or more of the respondents rated a 4 or 5 . A tenth category, Communication, which received high ratings by 49 percent of the respondents, also was included. An $X$ represents a mean score of 4 or higher for the depart- mental area's interest in a given category. From the table, it can be seen that the library departmental areas that expressed the highest interest in these ten categories are Administration (five categories), Cataloging (seven categories), and Circulation (five categories). Technology was the only category for which high interest encompassed a majority of departmental areas.

Among the other categories in the survey which had less overall interest, there were seven for which an individual departmental area indicated high interest (mean of 4 or higher). Not surprisingly, Media Services indicated a high interest in Macintosh Training and Software, and Copyright from the specialinterest group of categories. Spreadsheet training ranked high by Administration, and this information was used in scheduling workshops in this category to maximize the ability of personnel in this area to attend. Work Relations among Library Personnel was ranked high by Acquisitions, Personnel Leadership by Administration, and Email Basics by Administration and Acquisitions. Workshops have not yet been planned for these categories. Knowl- 


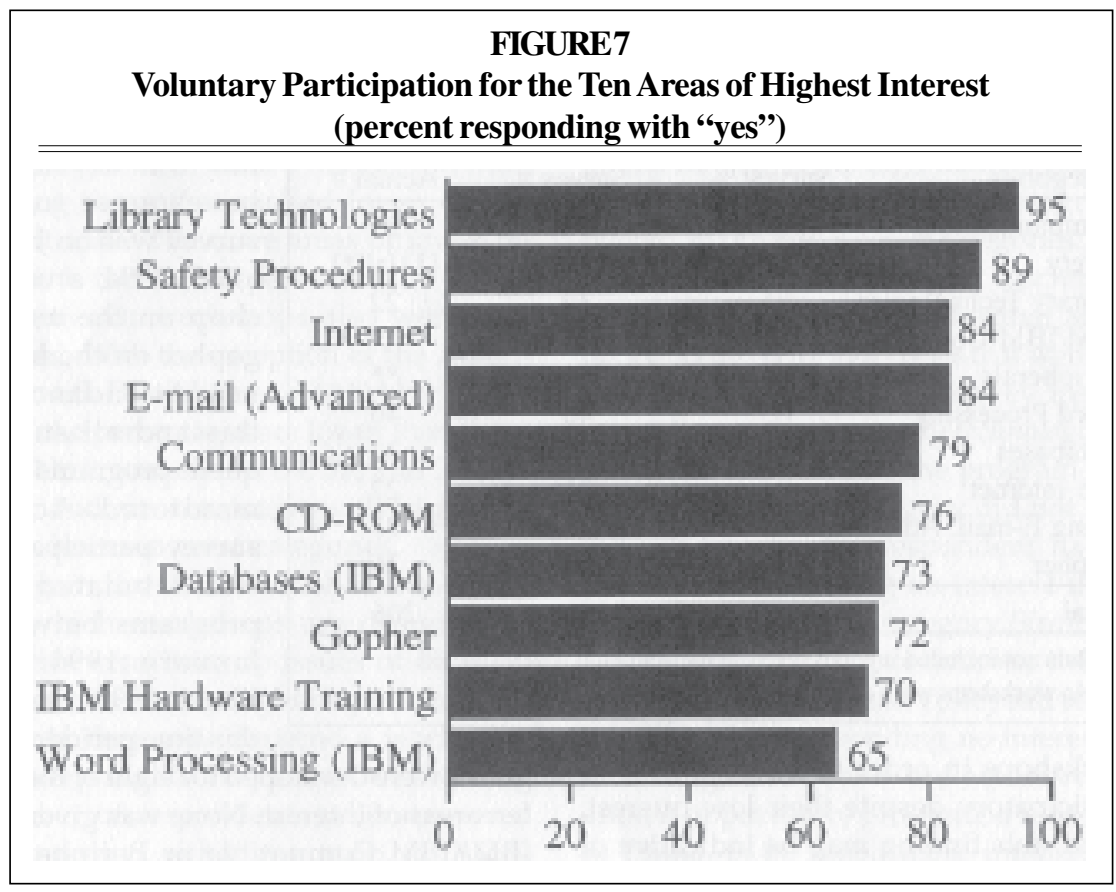

edge of these individual departmental needs will assist the committee in scheduling activities and tailoring activities that more effectively meet departmental needs.

\section{Programs and Voluntary Participation}

The percent of respondents who indicated voluntary participation in the ten areas of highest interest is represented in figure 7 . Nine of these categories received high ( 4 or higher) ratings by 50 percent or more of respondents. The tenth category, Communications, received high ratings by 49 percent of respondents. Within the Personnel Programs group, most respondents indicated they would participate in development activities if offered. Three of the Personnel Programs categories (Library Technologies, Safety Procedures, and Communications among Library Personnel) are represented in figure 7 . Technology exceeded all other categories in that 95 percent of respondents indicated they would participate, Safety
Procedures was next with 89 percent, and Communications showed 79 percent. Library and electronic resources provided the other seven categories in figure 7, with three from E-mail and Internet and two each from Hardware Training and IBM/IBM-Compatible Software Training. None came from Macintosh-related categories, and none came from the special-interest group of categories.

It would be expected that as level of interest increased, so too would indicators of participation. However, this effect was not as apparent as the committee had anticipated. All topics within Personnel Programs had at least 53 percent of respondents indicate that they would attend related developmental activities. However, most of these items received only slightly better-than-moderate ratings (3.0-3.4) in terms of interest. For example, although only 15 percent of respondents gave Sexual Harassment a high rating for interest (4 or higher), 53 percent indicated they would 


\begin{tabular}{|lccc|}
\hline \multicolumn{4}{|c|}{$\begin{array}{c}\text { TABLE 2 } \\
\text { Workshop Participation in Top Ten } \\
\text { Categories of Interest }\end{array}$} \\
\hline \hline Categories & Survey \% & Survey \# & Actual \# \\
\hline Communications & $79 \%$ & 30 & 22 \\
Safety & 89 & {$[32 *]$} & {$[11 /$ all*] } \\
Library Technologies & 95 & 39 & 22 \\
IBM/IBM-Compatible & $* *$ & $* *$ & $* *$ \\
Peripherals & $* *$ & $* *$ & $* *$ \\
Word Processing & 65 & 40 & 40 \\
Databases & 73 & 27 & 32 \\
The Internet & 84 & 31 & 27 \\
Using E-mail: Adv. & 84 & 32 & 27 \\
Gopher & 72 & 26 & 36 \\
\hline Total & \multicolumn{4}{|c}{225} \\
\hline *Data not included in totals because participation was restricted \\
** No workshops were offered in this category. \\
\hline
\end{tabular}

an individual from participating.

Level of interest more closely corresponded to indicators of participation for hardware and software issues ( $\mathrm{Li}$ brary and Electronic Resources). For example, all categories pertaining to Macintosh hardware and software received low percentages of high ratings. Accordingly, the majority of respondents indicated they would not at-

attend a developmental activity on this topic. This result surprised the committee members because it was thought that time commitments and work overloads would cause respondents to indicate intent to participate only in activities for which their interest was highest.

Three plausible explanations are given for this finding. First, a respondent, although not personally interested in a topic, may feel that obtaining knowledge about it through a workshop is beneficial for professional reasons. Second, personnel may feel obligated to attend workshops in order to be perceived as participatory despite their low interest. Third, this finding may be indicative of employees' perception of personnel development and training. Often there is a propensity to view training as a discretionary extra not directly linked to daily work activities. "This perception is reinforced by the belief that training comprises 'going on courses' and the idea that it is a day off (or days off) from work; a close relative of annual leave or time off for good behaviour."11 Therefore, low interest in a developmental activity does not necessarily preclude

tend a developmental activity related to these categories. For other categories in Library and Electronic Resources, higher-interest ratings were, in general, reflected in higher indicators of participation.

\section{Attendance Results for Programs Developed}

The first program that was developed in response to the survey was on January 7,1994, a workshop on the use of gopher on the Internet. Attendance at this and all subsequent programs was monitored. Actual survey participation was tabulated for programs between January 1994 and March 1995. During this time period, programs were developed for eight of the top ten areas of interest. None was given for IBM/IBM Compatible or Peripherals. Table 2 shows the actual number of participants for each of these eight categories. For purposes of comparing actual to predicted participation, this table also gives the number of survey respondents who indicated willingness to participate for each of the eight categories. The number in the first column in table 2 is the percent of respondents that the number from the survey (second column) represents. For example, thirty of the 
respondents who answered they would attend a workshop on communications (among library personnel) represents 79 percent of the total number of respondents who answered the question, either yes or no, for Communications.

The number of respondents predicted to participate from the survey and the actual number of respondents were each totaled, and the percent of actual (206) to predicted (225) participants was calculated to be 92 percent. Two activities were conducted in the area of Safety, a CPR training for which participation was limited to eleven, chosen to represent different areas of the library, and a tornado and fire drill, for which participation was 100 percent of those present in the library. These numbers are not included in the totals at the bottom of table 2 .

Table 3 is similar to table 2 except that the categories in table 3 are chosen from among those that ranked lower on the Likert scale. These are areas of lower interest to the library personnel, for which activities or workshops also were conducted. With the exception of the Americans with Disabilities Act (ADA) at 76 percent, the percentages are lower than those in table 2, namely 54, 54, 53, and 27 percent, and are consistent with responses from lower-interest categories.

Again, for ADA, which is a category for which workshops are designed to provide sensitivity to issues of disabled personnel, twenty-eight respondents answered they would attend a workshop. This represents 76 percent of the total number of respondents who answered the question, either yes or no, for ADA. The response of 76 per- cent for ADA in table 3 is more representative of the percentages indicated for the top ten areas of interest in table 2 , even though the level of interest, as represented by the Likert scale mean rating of 3.2, was relatively low. The higher-participation indication for ADA may show that some personnel felt they should attend, even though their level of interest was lower. In the end, eighteen people attended the workshop.

The number of respondents predicted to participate from the survey and the actual number of respondents was each totaled in table 3 , and the percent of actual (71) to predicted (91) participants was calculated to be 78 percent, as compared to 92 percent for the higher-interest categories in table 2 .

The mean value of the actual number of participants is twenty-nine for the high categories in table 2, as compared to fourteen for the low-interest categories in table 3. Overall, it can be said that there was nearly twice the attendance at programs offered in the high-interest categories as there was attendance at programs offered in the low-interest categories. It is worth noting that the largest discrepancies between predicted and actual attendance occurred for categories that were not as clearly defined in the survey instrument. For example, library technologies is a very broad term, and the program that was offered in this category did not necessarily fit what the respondent had in mind when indicating an interest in attending events in this category. Simi-

\section{TABLE 3}

\section{Workshop Participation in Categories of Lower Interest}

\begin{tabular}{lccc}
\hline Categories & Survey $\%$ & Survey \# & Actual \# \\
\hline ADA & $76 \%$ & 28 & 18 \\
Time Management & 54 & 21 & 10 \\
Spreadsheets & 54 & 19 & 13 \\
Promotion of Research & 53 & 16 & 13 \\
Recreational Opportunities & 27 & 7 & 17 \\
\hline Total & & 91 & 71 \\
\hline
\end{tabular}


larly, for Recreational Opportunities, the respondent may have had volleyball teams in mind when responding no interest in attending, and found the actual recreational opportunity program, a film visit to China, to be much more interesting. On the other hand, categories such as Word Processing, Databases, and the Internet were more specific, and there is closer correspondence between attendance predicted by the survey and actual attendance. More careful wording for the categories will likely make the results more meaningful.

\section{Conclusions and Recommendations}

The survey of personnel was useful to determine the areas of needs and interests and to assess the likelihood of high attendance at programs developed by the Personnel Development Committee. After the survey results were analyzed, the committee developed a series of programs that addressed the ten areas of most interest (see figure 7 ), and implemented alternative methods of addressing other issues where needs were expressed, such as dispensing information to library personnel using the e-mail listserv for Booth Library. There was some difficulty in developing programs because, in some cases, the category title was not specific enough to accurately determine the interest of the personnel. It is recommended that survey instruments be carefully designed with the end results in mind. One or more committee members should complete the survey and note changes or clarifications needed. Categories should be carefully worded and explained so that the meaning of the results will be clear.

The survey permitted evaluation of the following two objectives: (1) to determine if there was a relationship between personnel interest in a topic and personnel participation in programs developed, and (2) to determine if personnel interest and needs differ departmentally. The methods used appeared to be effective in identifying the needs of library personnel. From the data collected, it seems that personnel participation was influenced by program type. Topics related to such things as hardware and software, which have direct application to work responsibilities, had high-interest ratings and high indicators of participation. Hardware and software topics that did not have direct implications for work responsibilities had low-interest ratings and low indicators of participation.

This was not the case for personnel program categories. Although many respondents had low interest in topics, they had high indicators of participation. Personnel may feel increased knowledge about a topic is beneficial for professional reasons, or they may feel obligated to participate even though they lack interest. Moreover, there may be a tendency among personnel to view developmental activities as time off or compensation for working hard.

As expected, department interest in categories differed. For the committee's purpose, knowledge of these differences is useful for scheduling workshops. It also helps to ensure that programs developed are tailored to departmental needs. It is recommended that the survey instrument be designed to gather information about the respondent, such as his or her department and possibly faculty or staff status.

A comparison of survey results and actual participation (tables 2 and 3 ) shows that for the programs developed during this time period, indicators of both interest and willingness to participate were valid indicators of actual participation. It is recommended that indication of participation be included in the design of the survey.

\section{Epilogue}

As of this writing, all but one of the sixtytwo full-time personnel at Booth Library have participated in at least one of the 
programs offered since January 1994. Committee members have found that the survey continues to be a viable tool in planning and scheduling the educational activities of Booth Library's personnel development program.

\section{Notes}

1. Ronald G. Leach and Maureen Sullivan, "Staff Development at Indiana State University: Providing the Competitive Edge," Library Administration \& Management 4 (summer 1990): 138-40.

2. Thomas W. Shaughnessy, "Staff Development in Libraries: Why It Frequently Doesn't Take," Journal of Library Administration 9, no. 2 (1988): 5-12.

3. Vivienne Monty, "Effective Team Building and Personality Types," Special Libraries 85 (winter 1994): 1-7.

4. Leach and Sullivan, "Staff Development at Indiana State University," 138-40.

5. Michael Albert, "HR Profit Power," Personnel 67 (Feb. 1990): 47-49.

6. Daniel Mitchell and Mahmood Zaidi, "Macroeconomic Conditions and HRM-IR Practice," Industrial Relations 29 (spring 1990): 164-88.

7. Monty, "Effective Team Building and Personality Types," 1-7.

8. Patrick Noon, "Starting from Scratch: Developing a Staff Training and Development Programme in an Academic Library," Personnel Training and Education 9, no. 3 (1992): 65-71.

9. Patricia Weaver-Meyers, "ARL Libraries and Staff Development: A Suggested Model for Success," College E Research Libraries 51 (May 1990): 251-65.

10. Kathleen Low, "Computers, Libraries, Staff Development and Training: A Symbiotic Relationship," Library Software Review 10 (July-Aug. 1991): 279-80.

11. Noon, "Starting from Scratch," 65-71. 